\title{
Bioengineered Zinc Oxide Nanoparticles for Anti-Oxidant Applications
}

\author{
Subburaman Chandramohan, Dhanuskodi Saranya, Krishnan Sundar, Azhaguchamy Muthukumaran
}

\begin{abstract}
Zinc oxide nanoparticles find its versatile application in medicine and industry when compared with other metal nanoparticles. The present study focuses on biogenic synthesis of Zinc Oxide nanoparticles (ZnONPs) using lemon juice extract. Lemon juice extracts contains natural alkaloids and flavonoids which acts as reducing agent for the synthesis of nanoparticles. The synthesized zinc oxide nanoparticle is characterized by Scanning Electron Microscopy (SEM) and FTIR. FTIR results showed the presence of functional groups which represents ZnONPs and reducing groups responsible for the reduction. The SEM observation reveals the synthesized ZnONPs are cube shaped. The obtained nanoparticle was further studied for toxicity using zebrafish embryos. Toxicity results suggested that the toxicity is dose dependent. Malformations in the embryos such as yolk sac edema and twisted tail were seen in higher concentrations. The antioxidant activity of the synthesized ZnONPs was carried out using DPPH assay. Results implied that the antioxidant activity was also concentration dependent.
\end{abstract} assay

Keywords- ZnONPs, lemon juice extract, Toxicity, DPPH

\section{INTRODUCTION}

$\mathrm{Z}$ inc oxide nanoparticles finds its applications in numerous fields including information technology, agriculture, textile industries for stain proof, and for energy production in fuel cell, chemical sensors, coating and paints, by its attracting properties such as piezoelectric, pyroelectric and semiconducting properties. Because of their large band gap and higher excitation energy, it has efficient UV-blue emission [1] [2]. Zinc oxide is considered to be a 'GRAS' (generally recognized as safe) substance by the FDA. ZnONPs has two decades of its usage in various medicinal sectors notably antimicrobial, antiviral implications,

anticancer therapy, tissue engineering, imaging, drug delivery, diagnostics and bio-interfaces. The synthesizing methods of metal nanoparticles are available in physical, chemical and biological [3], hydrothermal, sol-gel, vapor phase process, microwave-assisted, direct precipitation and sono-chemical method. In recent years, biological synthesis of metal oxide nanoparticles gained more attention in which the microorganisms, plants extracts are used as stabilizers to

Revised Manuscript Received on December 15, 2019.

Dhanuskodi Saranya, Department of Biotechnology, K.S.R Engineering college,Trichengode, tamilnadu 637215, India. Email: saranyadhanuskodibt98@gmail.com

Subburaman Chandramohan, Department of Biotechnology, Kalasalingam Academy of Research and Higher Education, Krishnankoil, Tamil Nadu 626126, India. Email: chandramohan@klu.ac.in

Krishnan Sundar, Department of Biotechnology, Kalasalingam Academy of Research and Higher Education, Krishnankoil, Tamil Nadu 626126, India. Email: sundarkr@klu.ac.in

Azhaguchamy Muthukumaran*, Department of Biotechnology, Kalasalingam Academy of Research and Higher Education, Krishnankoil, Tamil Nadu 626126, India. Email: a.muthukumaran@klu.ac.in control the crystal growth and thereby minimizing its toxicity. Thus, in this study nanoparticles are synthesized by citrus juice of lemon extract and undergone toxicological analysis indicating the effects of ZnONPs in Zebra fish (Danio rerio).

Several researches have concentrated on the environmental impacts of various types of nanoparticles. However, only limited toxicological data is available about the effects of Nanoparticles on the aquatic and terrestrial ecosystem. Probably, nanoparticles may pollute the aquatic environment from unexpected releases on transportation, disposal operations and in the course of its production. Nanoparticles might cause damage to aquatic organisms, and could also become dangerous to humans through food web. Recent studies on toxicity of aqueous nanoparticles reveals the serious concern about the threats to green algae [4], fish [5] and crustaceans [6] and copepods [7] Since, Zebra fish possesses significant advantages such as rapid breeding, $85 \%$ similarity to the human genome and small size. Hence in this study, toxicity is examined by varying concentrations of ZnONPs and its impact on hatching, survival, heart beat and any notable malformations in embryo and larvae of the Zebra fish. The potential of ZnONPs in free radical scavenging antioxidant activity by DPPH assay are performed.

\section{MATERIALS AND METHODS}

\section{A. Nanoparticle synthesis and purification:}

$0.1 \mathrm{~g}$ of zinc acetate was dissolved in $50 \mathrm{ml}$ of distilled water by continuous stirring to prepare Zinc acetate stock solution. Lemon extract $(50 \mathrm{ml})$ was mixed with the zinc acetate stock solution. The solution mixture was autoclaved at $121^{\circ} \mathrm{C}$ for 20 minutes. The sample was cooled down to the room temperature and centrifuged at $10,000 \mathrm{rpm}$ for 10 minutes. The centrifugation was repeated thrice for the purification of nanoparticle and agglomeration was prevented by sonicating the nanoparticles. Finally, the pellet was suspended in $5 \mathrm{ml}$ of distilled water and made to dry in petri dish for 18 hours and the $\mathrm{ZnONPs}$ were collected in the Eppendorf tubes.

\section{B. Characterization of nanoparticle:}

The synthesized ZnONPs nanoparticles from the lemon juice extract was analyzed for the presence of functional groups using Fourier Transform Infrared Spectrophotometer within the range of $400 \mathrm{~cm}-1$ to $4000 \mathrm{~cm}-1$. (IR Tracer, shimadzu, Japan) by ATR (kbr pellet) method [8]. The morphological characteristics of the ZnONPs were measured using a Scanning Electron Microscope (EVO-18 Bruker, Germany).

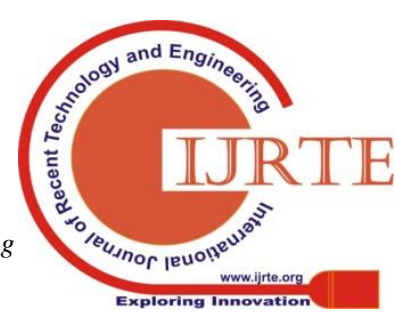




\section{Bioengineered Zinc Oxide Nanoparticles for Anti-Oxidant Applications}

Selected areas within the SEM sections were scanned for elemental composition analysis using EDX (Bruker 6130, Germany) coupled with SEM.

\section{Zebrafish husbandry and breeding:}

Culturing of zebrafish, breeding, embryo collection and embryonic larvae culture were performed according to the standard procedures. Briefly, adult fish were raised in a tank system with 12 hour alternating light/dark cycles, and Tubifex worms (free-dried) were fed twice a day. Mature, male and female zebrafish (at a ratio of 1 male to 2 females) were transferred in to a breeding tank and separated by a glass plate before breeding. All fertilized embryos were examined under an optical microscope and embryos with normal development were selected for embryo bioassay.

\section{Embryo and larval bioassays:}

Stock solution of ZnONPs was prepared by dissolving ZnONPs in vertex mixture and the intact $24 \mathrm{hpf}$ (hour post fertilized) zebra fish embryo or early blastula staged embryo were selected by an optical microscope for the study. The embryos were washed repeatedly with distilled water to remove dirt and debis on the surface of the embryo before starting the experiment. ZnONPs $(1 \mathrm{ml})$ of varying concentration gradient ie., 20, 30, 40, $50 \mu \mathrm{g} / \mathrm{ml}$ was added in the petri dish constituting $9 \mathrm{ml}$ of distilled water with 20 embryos in it and was monitored periodically. The embryos and larvae were observed periodically (48, 72 and $96 \mathrm{hpf}$ ) under optical microscope and it was documented. The incidence of malformations was compared with different concentration groups that of control. The developmental malformations, survival rate, hatching rate (total number hatched / total number taken for experiment $* 100 \%$ ), and heart beat rate per minute were recorded for the period of (96-hour post fertilization) 3 days.

\section{E. Antioxidant activity:}

The DPPH (1, 1- diphenyl-2-picrilhydrazyl) assay was performed for measuring free Radical Scavenging Activity of ZnONPs. Briefly, $1 \mathrm{ml}$ of ZnONPs of varying concentration $(20,30,40$ or $50 \mu \mathrm{g} / \mathrm{mL})$ was added to $1 \mathrm{ml}$ of $1 \mathrm{mM} \mathrm{DPPH}$ solution in a test tube respectively. Methanol was kept as blank solution and a test tube with $1 \mathrm{ml}$ of ascorbic acid the standard antioxidant with one $1 \mathrm{ml}$ of $1 \mathrm{mM}$ DPPH assay solution to calculate the free radical scavenging activity. The solutions were allowed to stand for 1 hour in the dark to protect from the light radiation. At the end of 1 hour, the reduction of the DPPH radical was determined by measuring the absorbance at $517 \mathrm{~nm}$. The free Radical Scavenging Activity was calculated by using the following equation:

$\%$ RSA $=[$ (absorbance of control - absorbance of sample)/absorbance of control] $\times 100$ [9]

Were, absorbance of control was the DPPH solution and absorbance of sample was the absorbance of the solution when the ZnONPs is added with the DPPH.

\section{RESULTS AND DISCUSSION}

\section{A. Characterization of synthesized ZnONPs.}

FTIR of the lemon extract mediated ZnONPs showed the formation of bands due to $\mathrm{OH}$ stretching around $3267 \mathrm{~cm}^{-1}$, $\mathrm{C}=\mathrm{C}$ group around $1600 \mathrm{~cm}^{-1}$. The peaks around $1600 \mathrm{~cm}^{-1}$ denotes citric acid which was responsible for the reduction of $\mathrm{ZnO}$ reaction. $420.48 \mathrm{~cm}^{-1}$ showed the Zinc finger print region (fig. 1).

SEM observation of the synthesized ZnONPs showed cubic shaped ZnONPs. SEM observation also showed that, there was no agglomeration of the nanoparticles synthesized and the average size of cubes were found to be around 150-200 nm.

\section{B. Effect of ZnONPs on zebrafish embryos}

Zebra fish embryos were treated with different concentrations of ZnONPs $(20,30,40 \& 50 \mu \mathrm{g} / \mathrm{ml})$ and their survival percentage to the respective concentration were shown on the (fig. 3). The graph revealed that there was a concentration dependent decrease in survival rate. Thus, the mortality rate increased with the increasing concentration. The concentration ranging from 40 and $50 \mu \mathrm{g} / \mathrm{ml}$ showed $80 \%$ and $75 \%$ survival rates respectively. The obtained results were similar to previous toxicity studies of ZnONPs [10]. Generally, ZnONPs have very low toxicity, and the biogenic synthesis is still found to have less toxicity.

The impingement of the ZnONPs in the hatching rate of the zebra fish embryos was also evaluated. (Fig. 4) showed the hatching rate of treated embryo at respective concentration of $\mathrm{ZnONps}$ and control. It was noticed that in high concentration (40 \& $50 \mu \mathrm{g} / \mathrm{ml}$ ) delayed in hatching. Thus, $33.33 \%$ of hatching lag compared with control was observed in concentration of 40 and $50 \mu \mathrm{g} / \mathrm{ml}$.

The heart beat details of zebra fish larvae exposed to ZnONPs at different concentrations was evaluated (fig. 5). There was a concentration dependent decrease in the heartbeat was noted The variation in heart beat rate were noted in different concentrations and compared with that of control.

The (fig. 6) shows the malformations which increased with higher concentration of ZnONPs (40 \& $50 \mu \mathrm{g} / \mathrm{ml})$. The observed malformations were embryo sac edema and twisted tail in the larvae (fig.7). In the doses of $20 \mu \mathrm{g} / \mathrm{ml}$ and $30 \mu \mathrm{g} / \mathrm{ml}$ no malformations were observed. Thus, ZnONPs at the concentration of $20 \mu \mathrm{g} / \mathrm{ml}$ and $30 \mu \mathrm{g} / \mathrm{ml}$ were considered highly safe. 


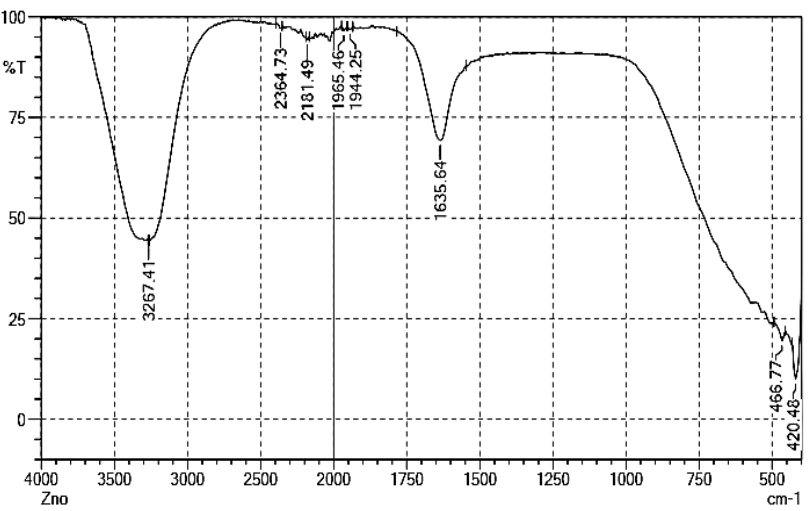

Fig.1.FTIR image of the synthesized ZnONPs.

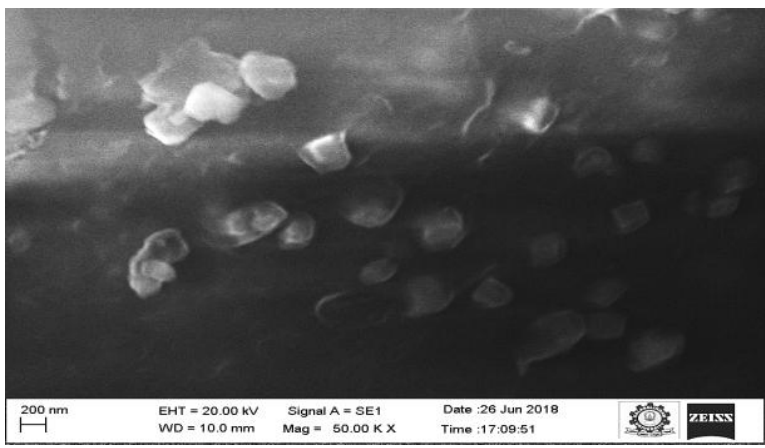

Fig.2.The SEM observation revealed the cube shaped ZnONPs.

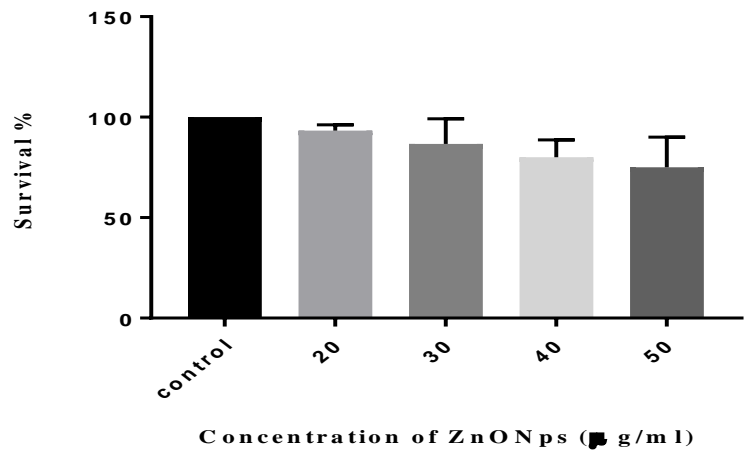

Fig.3.The graph representing the survival rate of the zebrafish embryo.

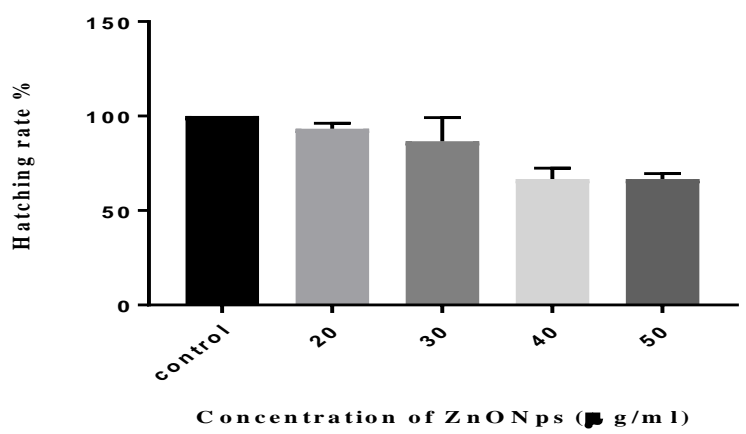

Fig.4.The graph representing the Hatching rate of the Zebrafish embryo.

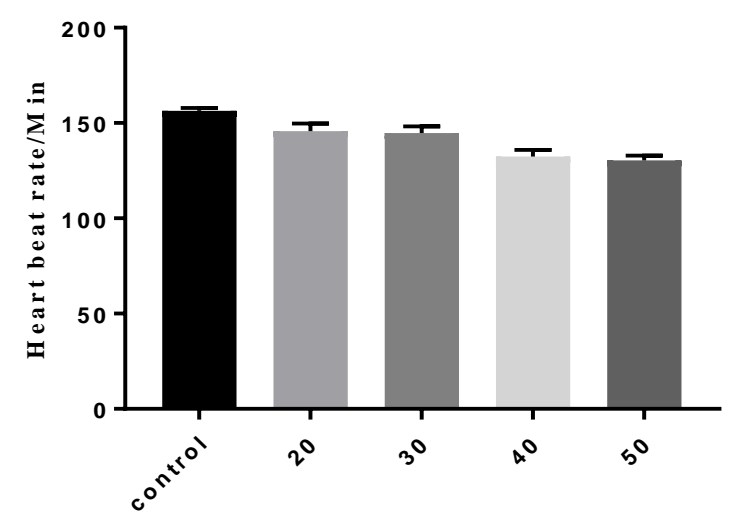

Concentration of ZnONps( g/m l)

Fig.5. The graph representing the Heart beat rate of the Zebrafish embryo.
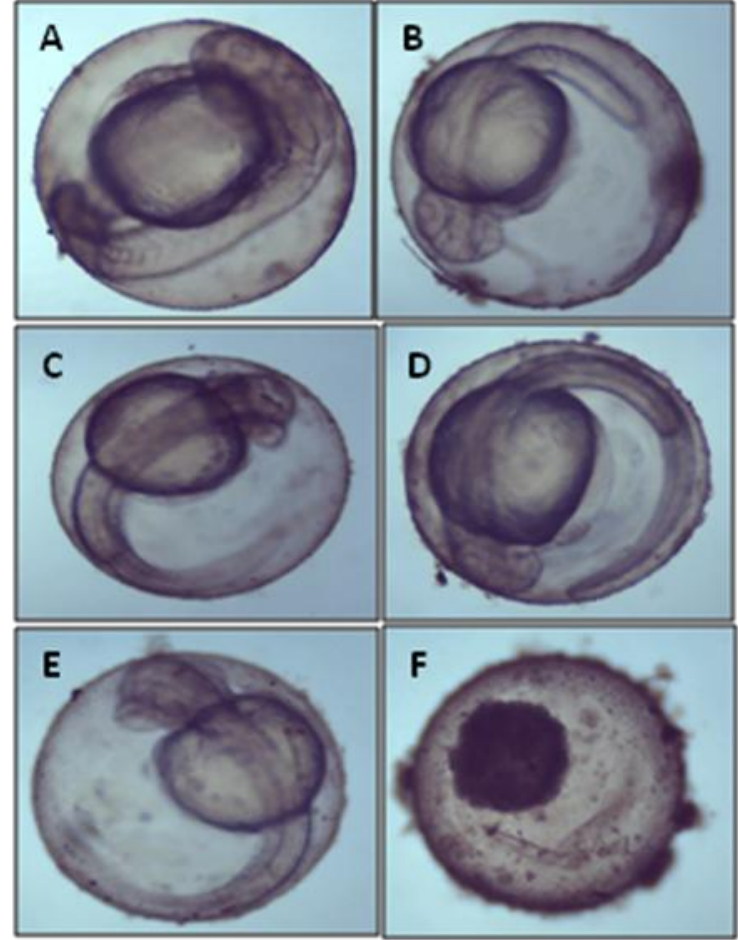

Fig.6. The microscopic observation of zebrafish embryos treated to various concentrations of ZnONPs. A \& B. control; C. $20 \mu \mathrm{g} / \mathrm{ml}$; D. $30 \mu \mathrm{g} / \mathrm{ml}$; E. $40 \mu \mathrm{g} / \mathrm{ml}$; F. 50 $\mu \mathrm{g} / \mathrm{ml}$.

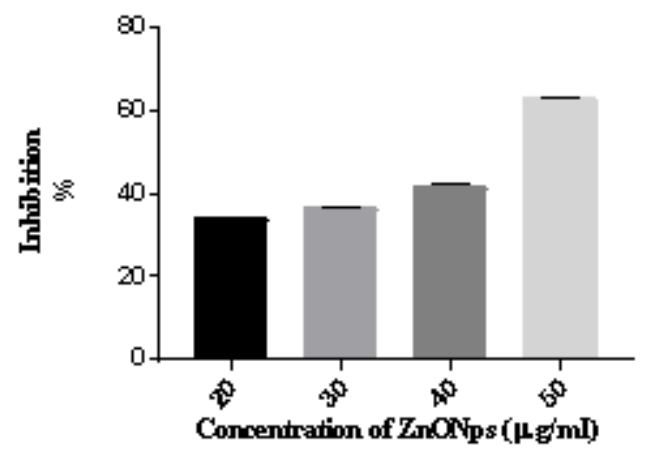

Fig.7.the graph elucidates the antioxidant activity percentage of $\mathrm{ZnONPs}$

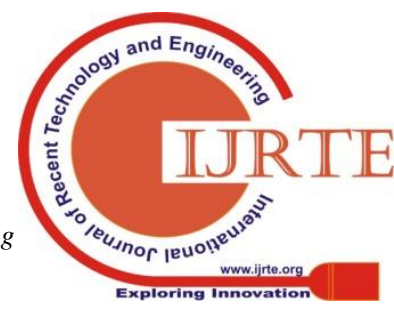




\section{Bioengineered Zinc Oxide Nanoparticles for Anti-Oxidant Applications}

\section{Antioxidant activity:}

Antioxidant activity of the Zinc oxide nanoparticle was recorded. There was a concentration dependent increase in antioxidant activity was seen. The antioxidant activity of the ZnONPs was performed by using DPPH assay. At $50 \mu \mathrm{g} / \mathrm{ml}$ concentration, the inhibition activity was found to be around $63 \%$.

\section{CONCLUSION}

The Biogenic synthesis of $\mathrm{ZnO}$ NPs from lemon juice extract was cost effective, possesses very low toxic effects. The synthesized nanoparticles remained stable for weeks without agglomeration. Lower concentrations of synthesized $\mathrm{ZnO}$ nanoparticles can be used in biomedical applications. The antioxidant activity of the lemon juice extract capped $\mathrm{ZnO}$ nanoparticles was also high in higher concentration of ZnONPs.

\section{ACKNOWLEDGMENT}

Authors are grateful to the Kalasalingam Academy of Research and Education for providing funding and research facilities.

\section{REFERENCES}

[1] M. Vafaee and M.S. Ghamsari, "Preparation and characterization of ZnO nanoparticles by a novel sol-gel route", Materials Letters, vol. 61, pp.3265-3268, 2007.

[2] Y. Zhang, Y. W. Tan, H.L. Stormer, and P. Kim, "Experimental observation of the quantum Hall effect and Berry's phase in graphene", nature, vol. 438, p.201. 2005

[3] S. Chandramohan, K. Sundar and A. Muthukumaran, "Monodispersed spherical shaped selenium nanoparticles (SeNPs) synthesized by Bacillus subtilis and its toxicity evaluation in zebrafish embryos", Materials Research Express, vol. 5, p.025020. 2018.

[4] K. Hund-Rinke and M. Simon, "Ecotoxic effect of photocatalytic active nanoparticles (TiO2) on algae and daphnids" Environmental Science and Pollution Research, vol. 13, pp.225-232, 2006.

[5] R.J Griffitt, R. Weil, K.A. Hyndman, N.D. Denslow, K. Powers, D. Taylor and D.S. Barber, "Exposure to copper nanoparticles causes gill injury and acute lethality in zebrafish (Danio rerio)", Environmental science \& technology, vol. 41, pp.8178-8186, 2007.

[6] P.J. Borm, D. Robbins, S. Haubold, T. Kuhlbusch, H. Fissan, K. Donaldson, R. Schins, V. Stone, W. Kreyling, J. Lademann and J. Krutmann, "The potential risks of nanomaterials: a review carried out for ECETOC". Particle and fibre toxicology, vol. 3, p.11, 2006.

[7] R.C. Templeton, P.L. Ferguson, K.M. Washburn, W.A. Scrivens, and G.T. Chandler, Life-cycle effects of single-walled carbon nanotubes (SWNTs) on an estuarine meiobenthic copepod, Environmental science \& technology, vol. 40, pp.7387-7393, 2006.

[8] L.A. Forato, R. Bernardes-Filho and L.A. Colnago, "Protein structure in $\mathrm{KBr}$ pellets by infrared spectroscopy", Analytical biochemistry, vol. 259, pp.136-141, 1998.

[9] S.P. Velammal, S.P, T.A. Devi and T.P. Amaladhas, "Antioxidant, antimicrobial and cytotoxic activities of silver and gold nanoparticles synthesized using Plumbago zeylanica bark", Journal of Nanostructure in Chemistry, vol. 6, pp.247-260, 2016.

[10] S. Jeyabharathi,K. Kalishwaralal, K .Sundar and A. Muthukumaran, "Synthesis of zinc oxide nanoparticles ( $\mathrm{ZnONPs}$ ) by aqueous extract of Amaranthus caudatus and evaluation of their toxicity and antimicrobial activity”,Materials Letters, 209, pp.295-298,2017.

\section{AUTHORS PROFILE}
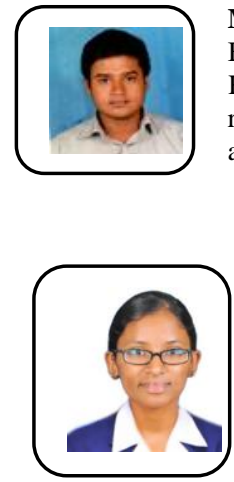

Ms. D. Saranya is currently doing he B.Tech-Biotechnolgy in K.S.R Engineering College, Tiruchengode.

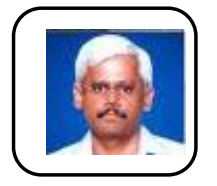

Dr. K. Sundar is currently working as senior Professor in the Department of Biotechnology and Dean in the School of Bio and Chemical engineering in Kalasalingam Academy of Research and Education. His research interests in Immunology and Vaccinology.

Dr. A. Muthukumaran is currently working as Associate Professor and Head in the Department of Biotechnology in Kalasalingam Academy of Research and Education. His research interests in Nanobiotechnology, Drug delivery and Safety assessment of nanoparticles 